\title{
DIGITALCOMMONS
}

—@WAYNESTATE-

Wayne State University

4-1-2007

\section{Suboptimal Minimax Design of Constrained Parabolic Systems with Mixed Boundary Control}

Boris S. Mordukhovich

Wayne State University, boris@math.wayne.edu

\section{Recommended Citation}

Mordukhovich, Boris S., "Suboptimal Minimax Design of Constrained Parabolic Systems with Mixed Boundary Control" (2007). Mathematics Research Reports. Paper 49.

http://digitalcommons.wayne.edu/math_reports/49

This Technical Report is brought to you for free and open access by the Mathematics at DigitalCommons@WayneState. It has been accepted for inclusion in Mathematics Research Reports by an authorized administrator of DigitalCommons@WayneState. 


\section{SUBOPTIMAL MINIMAX DESIGN OF CONSTRAINED PARABOLIC SYSTEMS WITH MIXED BOUNDARY CONTROL}

BORIS S. MORDUKHOVICH

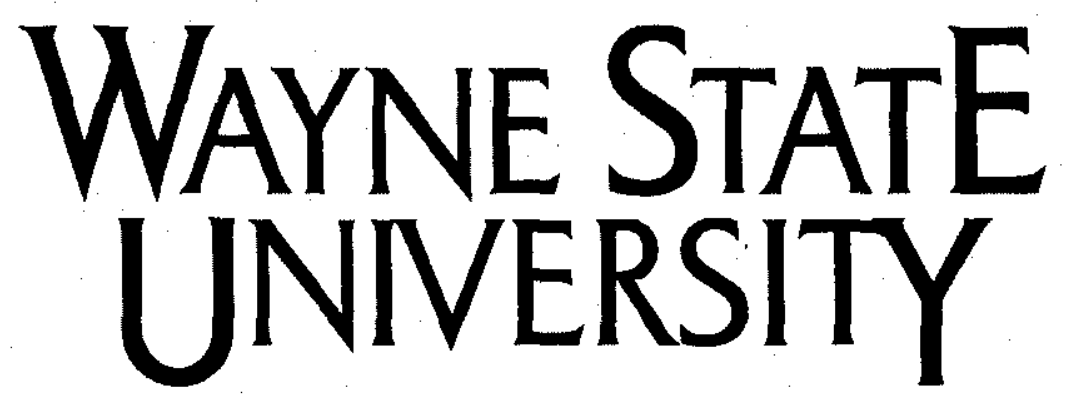

Detroit, MI 48202

Department of Mathematics

Research Report

2007 Series

\#4

This research was partly supported by the National Science Foundation and the Australian Research Council 


\title{
SUBOPTIMAL MINIMAX DESIGN OF CONSTRAINED PARABOLIC SYSTEMS WITH MIXED BOUNDARY CONTROL
}

\author{
BORIS S. MORDUKHOVICH ${ }^{1}$
}

\begin{abstract}
The paper concerns minimax control problems for linear multidimensional parabolic systems with distributed uncertain perturbations and control functions acting in mixed (Robin) boundary conditions. The main goal is to design a feedback control regulator that ensures the required state performance and robust stability under any feasible perturbations and minimize an energy-type functional under the worst perturbations from the given area. We design and justify an easily implemented suboptimal structure of the feedback boundary regulator and compute its optimal 'parameters ensuring the required state performance and robust stability of the nonlinear closed-loop control system on the infinite horizon.
\end{abstract}

Key words: parabolic systems, mixed boundary controls, state constraints, uncertain perturbations, feedback control, minimax synthesis, robust stability

\section{Introduction and Problem Description}

This paper is devoted to developing an efficient procedure of design a suboptimal feedback control regulator acting in the mixed/Robin boundary conditions of a multidimensional linear parabolic system with pointwise constraints on the state and control variables under uncertain perturbations. Problems of this type are among the most challenging in control theory while being among the most important for various applications. The original motivation for our development came from practical design problems of automatic control of the soil groundwater regime in irrigation engineering networks functioning under uncertain weather and environmental conditions; see [7] for technological descriptions and modeling. Further developments were motivated by the author's collaboration with the IIASA Dynamic Systems Program; see, e.g., $[10,12]$ and the references therein.

The system dynamics is given by the multidimensional linear parabolic equation

$$
\left\{\begin{array}{l}
\frac{\partial y}{\partial t}+A y=w(t) \quad \text { a.e. in } Q:=[0, T] \times \Omega \\
y(0, x)=0, \quad x \in \Omega \\
\left.\left(\alpha y+\frac{\partial y}{\partial \nu_{A}}\right)\right|_{\Sigma}=u(t), \quad \Sigma:=(0, T] \times \Omega
\end{array}\right.
$$

where $\alpha>0$ and $\frac{\partial}{\partial \nu_{A}}$ stands for the standard normal derivative on the boundary with respect to the operator $A$. Controls $u(\cdot)$ in (1.1) act in the mixed boundary conditions and distributed perturbations $w(\cdot)$ are on the right-hand side of the parabolic equation. In (1.1), $A$ is a self-adjoint and uniformly strongly elliptic operator on $L^{2}(\Omega)$ defined by

$$
A y:=-\sum_{i, j=1}^{n} \frac{\partial}{\partial x_{i}}\left(a_{i j}(x) \frac{\partial y}{\partial x_{j}}\right)-c y
$$

where $\Omega \subset \mathbb{R}^{n}$ is a bounded domain with the closure $\operatorname{cl} \Omega$ and the boundary $\partial \Omega$ that is supposed to be a sufficiently smooth $(n-1)$-dimensional manifold, and where $T>0$ is a

\footnotetext{
${ }^{1}$ Department of Mathematics, Wayne State University, Detroit, Michigan 48202, USA (boris@math.wayne.edu). This research was partly supported by the USA National Science Foundation under grants DMS-0304989 and DMS-0603846 and by the Australian Research Council under grant DP-0451168.
} 
fixed time bound. The sets of admissible controls $U$ and admissible perturbations $W$ are given, respectively, by

$$
\begin{aligned}
& U:=\left\{u \in L^{\infty}[0, T] \mid-\alpha \leq u(t) \leq \alpha \text { a.e. } t \in[0, T]\right\} \\
& W:=\left\{w \in L^{\infty}[0, T] \mid-\beta \leq w(t) \leq \beta \text { a.e. } t \in[0, T]\right\}
\end{aligned}
$$

with symmetric bounds given by some fixed numbers $\alpha>0$ and $\beta>0$.

Further, fix $x_{0} \in \Omega$ and suppose that we are able to collect information about the system performance $y\left(t, x_{0}\right)$ at this point. A crucial requirement on the system performance is to keep the motion $y\left(t, x_{0}\right)$ within the given distance $\eta>0$ from the initial equilibrium state $y(x, 0) \equiv 0$ for the whole dynamic process. This means imposing the pointwise state constraints

$$
-\eta \leq y\left(t, x_{0}\right) \leq \eta \text { a.e. } t \in[0, T]
$$

The main goal of boundary controls $u(\cdot)$ in (1.1) is to keep the motion $y\left(t, x_{0}\right)$ within the state constraints (1.5) for all admissible perturbations $w(\cdot)$ from (1.4). To do it, we have to design a feedback control regulator in the boundary conditions as a function of the state position $\xi=y\left(t, x_{0}\right)$. To formalize this procedure, consider a function $f: \mathbb{R} \rightarrow \mathbb{R}$ satisfying the summability condition

$$
|f(\gamma(t))| \in L^{1}[0, T] \text { whenever } \gamma(t) \in L^{2}[0, T]
$$

and construct boundary controls in (1.1) via the feedback law

$$
u(t):=f\left(y\left(t, x_{0}\right)\right), \quad t \in[0, T]
$$

We say that $f$ defines a feasible regulator if it satisfies (1.6), generates controls $u(\cdot) \in U$ by (1.7), and keeps the corresponding motions $y\left(t, x_{0}\right)$ within the prescribed constraint area (1.5) for every admissible perturbation $w \in W$ from (1.4). The set of all feasible regulators is labeled as $\mathcal{F}$.

To estimate the quality of feasible regulators $f=f(\xi)$, we consider the cost functional

$$
J(f):=\max _{w \in W}\left\{\int_{0}^{T}\left|f\left(y\left(t, x_{0}\right)\right)\right| d t\right\},
$$

which is an energy-type functional with respect to controls (1.7) in the boundary conditions of (1.1) subject to the symmetric constraints (1.3). The maximum operation in (1.8) reflects the required control energy needed to neutralize the adverse effect of the worst perturbations from (1.4). The minimax feedback control problem $(P)$ studied in this paper is as follows:

$$
\text { minimize } J(f) \text { over } f \in \mathcal{F} \text {. }
$$

It has been well recognized in control theory and applications that feedback control problems are the most challenging and important for any type of dynamical systems, while PDE systems provide additional difficulties and much less investigated in comparison, e.g., with the ODE dynamics; see more discussions and references in $[9,10]$. Furthermore, significant complications come from pointwise/hard constraints on control and (much more) 
state functions; the latter are of high nontriviality even for open-look control problems. We are not familiar with any device applicable to problem $(P)$ among a variety of approaches and results available in the theories of differential games, $H_{\infty}$-control, Riccati's feedback synthesis, etc.; see, e.g., $[2,3,4]$ and the references therein. In this paper we develop and significantly extend the approach to solving the feedback control problem $(P)$, which was initiated in [8] for the case of the one-dimensional heat equation in $(1.1)$; see $[9,10,12]$ for some results reported mostly for Dirichlet boundary controls.

Our approach is essentially based on certain underlying features of the parabolic dynamics, particularly on the monotonicity property of transients related to the fundamental Maximum Principle for parabolic equations. Due to this property, we are able to select the worst perturbations in the area (1.4) for the class of nonincreasing and odd feedbacks (1.7) and then to study the corresponding open-loop optimal control problem with pointwise state constraints as a reaction of the parabolic system to the worst perturbations. This eventually allows us to justify suboptimality of a three-positional feedback regulator $f=f(\xi)$ in (1.7) and compute its optimal parameters ensuring robust stability of the resulting nonlinear closed-loop control system. Details follow.

\section{Preliminaries}

Let $A$ in (1.2) be a self-adjoint and uniformly strongly elliptic operator on $L^{2}(\Omega)$, i.e., $c \in \mathbb{R}$ and the functions $a_{i j}: \mathrm{cl} \Omega \rightarrow \mathbb{R}$ satisfy the properties:

$$
\begin{aligned}
& a_{i j} \in C^{\infty}(\operatorname{cl} \Omega), \quad a_{i j}(x)=a_{j i}(x) \text { for all } x \in \Omega, \quad i, j=1, \ldots, n, \\
& \sum_{i, j=1}^{n} a_{i j}(x) v_{i} v_{j} \geq \nu \sum_{i=1}^{n} v_{i}^{2} \text { with some } \nu>0 .
\end{aligned}
$$

Observe that for all $(u, w) \in U \times W$ the parabolic system (1.1) admits a unique generalized solution $y=y(t, x) \in L^{2}(Q)$ in the sense of [6]. Consider the homogeneous boundary value problem

$$
\left\{\begin{array}{l}
-A \varphi+\lambda \varphi=0 \\
\left.\left(\alpha \varphi+\frac{\partial \varphi}{\partial \nu_{A}}\right)\right|_{\Sigma}=0
\end{array}\right.
$$

defining eigenvalues $\lambda$ and eigenfunctions $\varphi$. As well known (see, e.g., [1]), under the assumptions made there exists a sequence of solutions $\left\{\left(\lambda_{k}, \varphi_{k}\right)\right\}_{k \in \mathbb{N}}$ to $(2.2)$ such that

$\lambda_{k}=c k^{\frac{2}{n}}+o\left(k^{\frac{2}{n}}\right)$ for some $c>0, \quad\left\{\varphi_{k}\right\}_{k \in \mathbb{N}}$ is a complete orthonormal basis in $L^{2}(\Omega)$,

$\varphi_{k} \in C^{\infty}(\operatorname{cl} \Omega)$, and $\varphi_{k}$ are uniformly bounded for all $k \in \mathbb{N}$.

The following result [6] provides the basic spectral representation of solutions to (1.1).

Proposition 2.1 (spectral representation). Having $\left\{\left(\lambda_{k}, \varphi_{k}\right)\right\}_{k \in \mathbb{N}}$ from (2.2), denote

$$
\mu_{k}:=\int_{\Omega} \varphi_{k}(x) d x \text { and } \nu_{k}:=\int_{\Gamma} \varphi_{k}(\zeta) d \sigma_{\zeta}
$$

where $d \sigma_{\zeta}$ signifies the surface measure. Then given $(u, w) \in L^{2}[0, T] \times L^{2}[0, T]$, the corresponding unique solution $y \in L^{2}(Q)$ to $(1.1)$ admits the representation

$$
y(t, x)=\sum_{k=1}^{\infty}\left(\mu_{k} \int_{0}^{t} w(\theta) e^{\lambda_{k} \theta} d \theta+\nu_{k} \int_{0}^{t} u(\theta) e^{\lambda_{k} \theta} d \theta\right) e^{-\lambda_{k} t} \varphi_{k}(x)
$$


where the series strongly converges in $L^{2}(Q)$.

Employing next the classical Maximum Principle for parabolic equations [5] and a smooth approximation procedure similar to the proof of [10, Theorem 3.1], we get the following monotonicity property manifesting a crucial feature of the parabolic dynamics.

Proposition 2.2 (monotonicity property). Let $\left(u_{i}, w_{i}\right) \in U \times W, i=1,2$, be such that

$$
u_{1}(t) \geq u_{2}(t) \text { and } w_{1}(t) \geq w_{2}(t) \text { a.e. } t \in[0, T]
$$

and let $y_{i} \in L^{2}(Q), i=1,2$, be the corresponding generalized solutions to (1.1). Then

$$
y_{1}(t, x) \geq y_{2}(t, x) \text { a.e. }(t, x) \in Q .
$$

\section{Approximating Problems under Worst Perturbations}

We begin this section with clarifying the structure of the worst perturbations for feasible feedbacks $f \in \mathcal{F}$ in the minimax problem $(P)$. Confine our consideration by a class of feedbacks in (1.7) defined by nonincreasing and odd functions $f=f(\xi)$. This choice allows us to justify that for any feedback control the worst perturbations occur to be the extreme ones $w \equiv \beta$ and $w \equiv-\beta$.

Theorem 3.1 (worst perturbations). Suppose that a feasible feedback $f \in \mathcal{F}$ is a nonincreasing and odd function on $\mathbb{R}$. Then the worst perturbations $w(t)$ providing the maximum value to (1.8) over all $w \in W$ from (1.4) are the extreme ones from the admissible area:

$$
w \equiv \beta \text { and } w \equiv-\beta \text {. }
$$

Proof. We proceed similarly to the proof of [10, Theorem 3.2] given for Dirichlet boundary controls. The crucial steps include the convolution representation of the transients $\xi(t)=$ $y\left(t, x_{0}\right)$ established in [11] and the monotonicity property of Proposition 2.2; cf. [10] for more details.

Our next step is to consider problem $(P)$ under the worst perturbations (3.1). It is easy to observe from the full symmetricity in problem $(P)$ that it is sufficient to study the case of $w(t) \equiv \beta$ and $-\alpha \leq u(t) \leq 0$ a.e. $t \in[0, T]$ when we arrive at the following open-loop optimal control problem $(\bar{P})$ :

$$
\operatorname{minimize} J(u):=-\int_{0}^{T} u(t) d t
$$

along the parabolic system with the fixed perturbation

$$
\left\{\begin{array}{l}
\frac{\partial y}{\partial t}+A y=\beta \quad \text { a.e. }(t, x) \in Q \\
y(0, x)=0, \quad x \in \Omega \\
\left.\left(\alpha y+\frac{\partial y}{\partial \nu_{A}}\right)\right|_{\Sigma}=u(t), \text { a.e. }(t, x) \in \Sigma
\end{array}\right.
$$

subject to the pointwise control and state constraints on $u(\cdot) \in L^{\infty}[0, T]$ and $y\left(\cdot, x_{0}\right) \in$ $L^{2}[0, T]$ :

$$
-\alpha \leq u(t) \leq 0 \text { and } y(t):=y\left(t, x_{0}\right) \leq \eta \text { a.e. } t \in[0, T]
$$


Problem $(\bar{P})$ is a state-constrained mixed boundary control problem, which belongs to a challenging class of hard-constrained problems in PDE optimal control. Following [8, $10]$ in the case of Dirichlet boundary control, we develop now an efficient approach to solve $(\bar{P})$ based first on $O D E$ approximations of the parabolic system (3.3) and then on subsequent penalty-type approximations of state constraints. To proceed, we use the spectral representation

$$
y\left(t, x_{0}\right)=\sum_{k=1}^{\infty}\left(\frac{\mu_{k} \beta}{\lambda_{k}}\left(e^{\lambda_{k} t}-1\right)+\nu_{k} \int_{0}^{t} u(\theta) e^{\lambda_{k} \theta} d \theta\right) e^{-\lambda_{k} t} \varphi_{k}\left(x_{0}\right)
$$

of solutions to (3.3) at $x=x_{0}$ due to Proposition 2.1, where the series in (3.5) converges strongly in $L^{2}[0, T]$. Taking any natural $N=1,2, \ldots$, we replace series (3.5) by the finite $N$-sum

$$
y^{N}\left(t, x_{0}\right)=\sum_{k=1}^{N}\left(\frac{\mu_{k} \beta}{\lambda_{k}}\left(e^{\lambda_{k} t}-1\right)+\nu_{k} \int_{0}^{t} u(\theta) e^{\lambda_{k} \theta} d \theta\right) e^{-\lambda_{k} t} \varphi_{k}\left(x_{0}\right)
$$

for which $y^{N}\left(t, x_{0}\right) \rightarrow y\left(t, x_{0}\right)$ strongly in $L^{2}[0, T]$. Furthermore, it is easy to observe that $y^{N}\left(t, x_{0}\right)$ in (3.6) is represented as the $N$-sum of $y_{k}(t)$, which satisfy the corresponding $O D E$ :

$$
\dot{y_{k}}=-\lambda_{k} y_{k}+\varphi_{k}\left(x_{0}\right)\left(\mu_{k} \beta+\nu_{k} u(t)\right) \text { a.e: } t \in[0, T], y_{k}(0)=0 .
$$

Throughout the rest of the paper we impose the following standing assumptions:

(H) The first eigenvalue $\lambda_{1}$ in (2.2) and the corresponding weights $\mu_{1}$ and $\nu_{1}$ are positive.

It is well known from the classical PDE theory that all the properties in (H) hold if, e.g., the operator $-A$ is the Laplacian and if the domain $\Omega$ is of a symmetric form (ball, rectangular, etc.). Note also from $\mu_{1}>0$ that $\varphi_{1}(x)>0$ on some subset of $\Omega$ of a positive measure; we always assume in what follows that the point of observation $x_{0}$ belongs to the latter subset.

Due to $(H)$, the first term in (3.5) dominates the exponential series, which is the case of a sufficiently large time interval $[0, T]$ of the dynamic process. This allows us pay a special attention to the case of $N=1$ in (3.6) and (3.7) for determining an appropriate suboptimal control structure in $(\bar{P})$ and then for its implementation into the feedback control system. We refer the reader to [10] for more justifications on the first-order approximation in the case of Dirichlet boundary control, which can be similarly done in the case of mixed boundary control under consideration.

\section{Exact Solution to the First-Order Approximation}

In this section we provide a detailed study of the first-order approximation optimal control problem $\left(\bar{P}_{1}\right)$ : minimize the (3.2) over admissible pairs $(u, y)$ satisfying

$$
\dot{y}=-\lambda_{1} y+\mu_{1} \varphi_{1}\left(x_{0}\right)\left(\mu_{1} \beta+\nu_{1} u(t)\right) \text { a.e. } t \in[0, T], y(0)=0,
$$

subject to the pointwise control and state constraints (3.4). Observe that the presence of the pointwise state constraints in (3.4) places $\left(\bar{P}_{1}\right)$ among the most challenging problems 
of ODE control theory. Available optimality conditions for such problems involve Borel measures that make them very difficult for verification, implementation, and applications.

We develop a different approach to solve $\left(\bar{P}_{1}\right)$, which employs a penalty-type procedure to approximate state constraints, then deals with solving approximating problems in the absence of state constraints, and finally derives optimal solutions to the state-constrained problem $\left(\bar{P}_{1}\right)$ by passing to the limit from optimal solutions to the approximating problems.

This approach occurs to be highly efficient for the class of problems under consideration. It allows us to find exact optimal solutions to the approximating problems based on the Pontryagin maximum principle [13], which provides necessary and sufficient optimality conditions for these problems, and then to compute by passing to the limit the exact optimal control to the constrained problem $\left(\bar{P}_{1}\right)$. It surprisingly happens that the optimal control for the state-constrained problem enjoys a simpler structure in comparison with the unconstrained approximating problems, and that overall the pointwise state constraint (3.4) turns out to be a regularization factor in this setting.

The following theorem provides an exact solution to the state-constrained problem $\left(\bar{P}_{1}\right)$. In the case when the time interval $T$ is sufficiently large, the optimal control obtained in this theorem is two-positional combining bang-bang and singular parts, with no measure involved.

Theorem 4.1 (exact solution to the state-constrained ODE optimal control problem). Let $\mu_{1} \varphi_{1}\left(x_{0}\right) \beta>\lambda_{1} \eta$. Assume in addition that

$$
\text { either } \quad \tau:=\frac{1}{\lambda_{1}} \ln \frac{\mu_{1} \varphi_{1}\left(x_{0}\right) \beta}{\mu_{1} \varphi_{1}\left(x_{0}\right) \beta-\lambda_{1} \eta} \geq T, \quad \text { or } \quad \mu_{1} \varphi_{1}\left(x_{0}\right) \beta-\alpha \nu_{1} \varphi_{1}\left(x_{0}\right) \leq \lambda_{1} \eta
$$

Then system (4.1), (3.4) is controllable, i.e., there is an admissible control $u(\cdot)$ that generates the trajectory $y(\cdot)$ satisfying the state constraint. Furthermore, an optimal control to $\left(\bar{P}_{1}\right)$ is given by

$$
\bar{u}(t)= \begin{cases}0 & \text { if } t \in[0, \bar{\tau}), \\ \frac{\lambda_{1} \eta-\mu_{1} \varphi_{1}\left(x_{0}\right) \beta}{\nu_{1} \varphi_{1}\left(x_{0}\right)} & \text { if } t \in[\bar{\tau}, T]\end{cases}
$$

where $\bar{\tau}:=\min \{\tau, T\}$ with $\tau$ computed in (4.2).

Proof. Observe that under the first condition in (4.2) the trajectory $y(t)$ of $(4.1)$ corresponding to the control $u(t) \equiv 0$ on $[0, T]$ satisfies the state constraint in (3.4). Therefore, in this case problem $\left(\bar{P}_{1}\right)$ admits the trivial optimal solution $u(t) \equiv 0$.

Assume now that the latter condition does not hold, i.e., $T$ is sufficiently large. Taking into account the second condition in (4.2), it is easy to check that the two-positional control (4.3) is feasible to $\left(\bar{P}_{1}\right)$. It remains to justify its optimality.

To furnish this, we introduce the following parametric family of approximating optimal control problem $\left(\bar{P}_{1 \varepsilon}\right)$ as $\varepsilon \downarrow 0$ :

$$
\text { minimize } J_{\varepsilon}(u):=\int_{0}^{T}\left(-u(t)+\frac{1}{\varepsilon}(\max \{0, y(t)-\eta\})^{2}\right) d t
$$

over measurable controls $u(\cdot)$ satisfying $-\alpha \leq u(t) \leq 0$ for a.e. $t \in T$ with no state constraints on trajectories to (4.1). It is well known [13] that the Pontryagin maximum principle 
provides necessary and sufficient conditions for optimal controls to $\left(\bar{P}_{1 \varepsilon}\right)$, which always exist for this kind of problems.

Employing this fundamental result and proceeding similarly to the proof of $[10$, Theorem 4.1] developed for the case of Dirichlet controls in the original parabolic problem, we arrive at the following exact optimal solution to each $\left(\bar{P}_{1 \varepsilon}\right)$ :

$$
u_{\varepsilon}(t)= \begin{cases}0 & \text { if } t \in\left[0, \tau_{1 \varepsilon}\right) \cup\left(\tau_{2 \varepsilon}, T\right], \\ \frac{\lambda_{1}\left(2 \eta \nu_{1} \varphi_{1}\left(x_{0}\right)+\lambda_{1} \varepsilon\right)}{2 \nu_{1}^{2} \varphi_{1}^{2}\left(x_{0}\right)}-\frac{\mu_{1} \beta}{\nu_{1}} & \text { if } \in\left[\tau_{1 \varepsilon}, \tau_{2 \varepsilon}\right]\end{cases}
$$

where the switching times $\tau_{1 \varepsilon}$ and $\tau_{2 \varepsilon}$ are computed by

$$
\begin{gathered}
\tau_{1 \varepsilon}=\frac{1}{\lambda_{1}} \ln \left[1-\frac{\lambda_{1}}{\mu_{1} \varphi_{1}\left(x_{0}\right) \beta}\left(\frac{\lambda_{1} \varepsilon}{2 \nu_{1} \varphi_{1}\left(x_{0}\right)}+\eta\right)\right]^{-1} \\
\tau_{2 \varepsilon}=T+\frac{1}{\lambda_{1}} \ln \left[1-\lambda_{1} \sqrt{\frac{\varepsilon}{\nu_{1} \varphi_{1}\left(x_{0}\right)\left(\mu_{1} \varphi_{1}\left(x_{0}\right) \beta-\lambda_{1} \eta\right)}}\right] .
\end{gathered}
$$

It is worth observing that the second (nonzero-actually intermediate between $-\alpha$ and 0) position in (4.5) is not a bang-bang position like the first one, but a characteristic of a singular mode, which cannot be found right from the Pontryagin maximum principle; see [10] for more details.

By passing to the limit in (4.5)-(4.7) as $\varepsilon \downarrow 0$, we get

$$
\tau_{1 \varepsilon} \downarrow \tau, \quad \tau_{2 \varepsilon} \uparrow T, \text { and } \int_{0}^{T} u_{\varepsilon}(t) d t \rightarrow \int_{0}^{T} \bar{u}(t) d t
$$

where $\tau$ and $\bar{u}(t)$ are given by (4.2) and (4.3), respectively. This easily implies the optimality of control (4.3) to $\left(\bar{P}_{1}\right)$ by the structures of the cost functionals (3.2) and (4.4) in the stateconstrained and approximation problems.

\section{Optimal Parabolic Control under Worst Perturbations}

In this section we address the open-loop optimal control problem $(\bar{P})$ involving the parabolic dynamic and pointwise state constraints, optimizing now the two-positional control structure whose suboptimality is justified above. Thus we arrive at the following problem $(\widehat{P})$ of dynamic optimization:

$$
\operatorname{minimize} J(v, \tau):=-\int_{0}^{T} u(t) d t
$$

over the parabolic system (3.3) with mixed boundary controls of the form

$$
u(t)=\left\{\begin{aligned}
0 & \text { if } t \in[0, \tau] \\
-v & \text { if } t \in(\tau, T]
\end{aligned}\right.
$$

subject to the constraints on control recourses $v$ and switching times $\tau$ given by

$$
0 \leq v \leq \alpha, \quad 0 \leq \tau \leq T
$$


together with the pointwise state constraints $y(t) \leq \eta$ on $[0, T]$.

The next theorem gives an exact optimal solution to problem $(\widehat{P})$, which therefore provides a suboptimal solution to the parabolic optimal control problem $(\bar{P})$ for all $T$ sufficiently large. Define the following aggregate spectral parameter of the operator $A$ :

$$
\gamma:=\sum_{k=1}^{\infty} \frac{\mu_{k} \varphi_{k}\left(x_{0}\right)}{\lambda_{k}} \text { and } \rho:=\sum_{k=1}^{\infty} \frac{\nu_{k} \varphi_{k}\left(x_{0}\right)}{\lambda_{k}}
$$

which are positive under the assumptions of this theorem.

Theorem 5.1 (optimal parameters of open-loop suboptimal control structure for the constrained parabolic system). In addition to the standing assumptions, suppose that

$$
0<\gamma \beta-\eta<\min \left\{\alpha \rho, \frac{\rho \mu_{1} \beta}{\nu_{1}}\right\}
$$

Then the following assertions hold:

(i) The transcendental equation

$$
\sum_{k=1}^{\infty} \frac{\varphi_{k}\left(x_{0}\right)}{\lambda_{k}} e^{-\lambda_{k} T}\left[\nu_{k}(\gamma \beta-\eta) e^{\lambda_{k} \tau}-\rho \mu_{k} \beta\right]=0
$$

has a unique solution $\tau=\bar{\tau}(T) \in(0, T)$ for all $T$ sufficiently large.

(ii) Any control in the form of (5.1) with

$$
v:=\frac{\gamma \beta-\eta}{\rho}
$$

is not only feasible to $(\widehat{P})$ for all positive $\tau \leq \bar{\tau}(T)$ but also optimal to this problem when $\tau=\bar{\tau}(T)$.

(iii) The solutions to (5.2) for all $T$ sufficiently large satisfy $\bar{\tau}(T) \downarrow \bar{\tau}$ as $T \rightarrow \infty$, where the asymptotically optimal switching time $\bar{\tau}$ is computed by

$$
\bar{\tau}=\frac{1}{\lambda_{1}} \ln \frac{\rho \mu_{1} \beta}{\nu_{1}(\gamma \beta-\eta)}
$$

Proof. Consider the trajectory $y(t, x)$ to (3.3) corresponding to the boundary control (5.1) and denote by $y(t):=y\left(t, x_{0}\right), 0 \leq t \leq \tau$ and $y(t ; \tau):=y\left(t, x_{0}\right), \tau \leq t \leq T$. By Proposition 2.1 we have

$$
\begin{gathered}
y(t)=\beta\left(\gamma-\sum_{k=1}^{\infty} \frac{\mu_{k} \varphi_{k}\left(x_{0}\right)}{\lambda_{k}} e^{-\lambda_{k} t}\right) \text { for } t \in[0, \tau] \\
y(t ; \tau)=(\gamma \beta-\rho v)+\sum_{k=1}^{\infty} \frac{\varphi_{k}\left(x_{0}\right)}{\lambda_{k}} e^{-\lambda_{k} t}\left[\nu_{k} v e^{\lambda_{k} \tau}-\mu_{k} \beta\right], \quad t \in[\tau, T] .
\end{gathered}
$$

Let $t=T_{0}>0$ be a solution to $y(t)=\eta$, which uniquely exists under the standing assumptions. When $T \leq T_{0}$, the control $u(t) \equiv 0$ on $[0, T]$ is obviously feasible and hence 
optimal to both problems $(\widehat{P})$ and $(\bar{P})$. In what follows we consider the case of $T>T_{0}$. Since

$$
y(t ; \tau) \rightarrow \gamma \beta-\rho v \text { as } t \rightarrow \infty
$$

every control from (5.1) stabilizes $y(t ; \tau)$ at the upper boundary $y=\eta$ of the state constraints. However, the controllability may be violated if $\tau$ is not properly selected in (5.1).

Employing the Maximum Principle for parabolic equations, we conclude from (5.6) and (5.7) that the optimal switching time $\bar{\tau}(T)$ is the maximum value of $\tau \leq T_{0}$, which ensures $y(t ; \tau) \leq \eta$ for all $t \in[\tau, T]$. Taking into account the monotonicity of transients in (5.7) with respect to $t$ and $\tau$, we obtain $\bar{\tau}(T)$ from the equation $y(T ; \bar{\tau}(T))=\eta$ with $v$ computed in (5.4). This reduces to (5.3). Using again the Maximum Principle, we conclude that $\bar{\tau}(T)$ is nonincreasing and thus converging. Its limit gives $\bar{\tau}$ in (5.5), which is the maximal switching moment in (5.1) ensuring the fulfillment of the state constraints on the infinite horizon $[0, \infty)$. The latter can be justified by applying the Fermat stationary rule to (5.7). $\triangle$

The results of Theorem 5.1 particularly demonstrate that the passage to the infinite horizon allows us to significantly simplify optimal solutions to the open-loop control problem under consideration, which reveals a certain turnpike property as $t \rightarrow \infty$.

\section{Feedback Suboptimal Control of Parabolic Dynamics}

The results obtained above and the full symmetry of the initial problem $(P)$ allow us to justify the following suboptimal structure $f=f(\xi)$ of feedback controls (1.7) in the parabolic system (1.1) with mixed boundary conditions:

$$
f(\xi)=\left\{\begin{array}{cl}
-v & \text { if } \xi \geq \sigma \\
0 & \text { if }-\sigma<\xi<\sigma \\
v & \text { if } \xi \leq-\sigma
\end{array}\right.
$$

This describes a three-positional regulator with the "dead region" $(-\sigma, \sigma)$. Observe that the three-positional feedback law $f(\xi)$ in $(6.1)$ is given by a nonincreasing and odd function satisfying the requirements of Theorem 3.1. The feedback control synthesis design reduces now to determining appropriate parameters $(v, \sigma)$ in $(6.1)$ such that the resulting closedloop control system keeps the state position $\xi=y\left(t, x_{0}\right)$ within the state constraint area (1.5) whatever uncertain perturbation $w \in W$ is realized and then ensures the minimum value of (1.8) under the worst perturbations.

The next theorem answers the above questions providing in fact the exact calculation of the optimal value $\sigma(T)$ on the given time interval $[0, T]$ and fully describes its limiting/asymptotic behavior as $T \rightarrow \infty$, which corresponds to problem $(P)$ on the infinite horizon.

Theorem 6.1 (feasible and optimal parameters of the three-positional regulator). Under the standing assumptions, let $v$ in (6.1) be computed by (5.4), and let

$$
\sigma(T):=\beta\left(\gamma-\sum_{k=1}^{\infty} \frac{\mu_{k} \varphi_{k}\left(x_{0}\right)}{\lambda_{k}} e^{-\lambda_{k} \tau(T)}\right)
$$


where $\tau(T)$ is the unique solution to (5.3) for $T$ sufficiently large. Then the feedback control (6.1) is feasible to $(P)$ on $[0, T]$ for all $0<\sigma \leq \sigma(T)$ being optimal to $(P)$ on this interval when $\sigma=\sigma(T)$. We further have $\sigma(T) \downarrow \bar{\sigma}$ as $T \rightarrow \infty$, where $\bar{\sigma}>0$ is computed by

$$
\bar{\sigma}:=\beta\left(\gamma-\sum_{k=1}^{\infty} \frac{\mu_{k} \varphi_{k}\left(x_{0}\right)}{\lambda_{k}}\left[\frac{\nu_{1}(\gamma \beta-\eta)}{\rho \mu_{1} \beta}\right]^{\frac{\lambda_{k}}{\lambda_{1}}}\right) .
$$

Moreover, the three-positional regulator (6.1) with $v$ computed in (5.4) is feasible to $(P)$ on $[0, \infty)$ whenever $0<\sigma \leq \bar{\sigma}$ being in fact optimal on the infinite horizon $[0, \infty)$ when $\sigma=\bar{\sigma}$.

Proof. Taking into account the three-positional structure in (6.1) and the monotonicity of transients to (1.1) with respect to perturbations, we conclude that (6.1) keeps the state constraints (1.5) for any $w \in W$ when it does this for the worst perturbations (3.1). Thus to find the optimal value $\sigma(T)$, we should maximize the width of the dead region for which the state constraints (1.5) hold in the worst/extreme case (3.1) on $[0, T]$.

It follows from the time monotonicity of the transient $y(t)$ in (3.3) that $\sigma(T)=y(\tau(T)$ ), where $\tau=\tau(T)$ solves (5.3). This justifies formula (6.2). Passing to the limit in (6.2) as $T \rightarrow \infty$, we arrive at the asymptotically optimal value (6.3). It follows from Theorem 5.1 and the above discussions that the latter control law keeps the state constraints (1.5) on the infinite interval $[0, \infty)$ for the worst perturbations, and thus for smaller perturbations as well. Therefore, the value of $\bar{\sigma}$ in (6.3) gives us the optimal/maximal width of the dead region ensuring the fulfillment of the state constraints (1.5) for all $w \in W$ on the infinite horizon. This completes the proof of the theorem.

\section{Robust Stability of the Closed-Loop Parabolic System}

Consider the closed-loop parabolic system withe the three-positional feedback regulator (6.1) in the mixed boundary conditions:

$$
\left\{\begin{array}{l}
\frac{\partial y}{\partial t}+A y=w(t), \quad x \in \Omega, \quad t \geq 0 \\
y(0, x)=0, \quad x \in \Omega \\
\left.\left(\alpha y+\frac{\partial y}{\partial \nu_{A}}\right)\right|_{\Sigma}=f\left(y\left(t, x_{0}\right)\right), \quad t \geq 0 .
\end{array}\right.
$$

Our goal is to derive efficient conditions ensuring the robust stability of system (7.1), (6.1) in the sense precisely defined below. Note that system (7.1) is highly nonlinear due to the discontinuous regulator (6.1) in the boundary conditions; so it may loose stability in the large of the initial equilibrium state $y \equiv 0$. Another major source of the possible loss of stability is that system (7.1) is of distributed parameters exhibiting the inertia/delay between control actions on the boundary and the current state position $\xi=y\left(t, x_{0}\right)$ at the intermediate point of observation $x_{0} \in \Omega$ of the space domain.

We are not familiar with any results on robust stability of systems like (7.1), except the recent ones obtained in [10] for the case of Dirichlet boundary conditions. To derive efficient conditions ensuring stability in the large of the equilibrium state $y \equiv 0$ in (7.1), we develop a variational approach based on monotonicity properties of the parabolic dynamics that reduces the stability issue to solving an open-loop optimal control problem for (1.1) on the infinite horizon. 
Observe from the structure of the closed-loop system (7.1) that the required robust stability can be lost if the dead region in (6.1) is not sufficiently wide. Indeed, in such cases the transients $\xi=y\left(t, x_{0}\right)$ would move back and forth between the dead region boundaries under switching control positions in (6.1) with no external perturbations, just by inertia of the control system. This means that the closed-loop control system (7.1), (6.1) may start functioning in a non-acceptable self-vibrating regime as $t \rightarrow \infty$ thus signifying instability in the large of the initial equilibrium state $y=0$. We intend to find conditions that exclude such instability.

It follows from the above discussion that the unstable self-vibrating regime will not occur if the transient $y\left(t, x_{0}\right)$ starting at one boundary of the dead region does not reach the other boundary whenever $t>0$ under the control switching in (6.1) with no external perturbations. Moreover, the limiting stability resource of the system relates to the minimal width of the dead region ensuring the afore-mentioned property. This allows us to derive efficient stability conditions by solving an open-loop optimal control problem for (1.1) on the infinite horizon.

Theorem 7.1 (robust stability). Let (7.1) be a closed-loop parabolic system under the standing assumptions made, and let (6.1) be a three-positional feedback regulator in the boundary conditions of (7.1) with arbitrary parameters $v>0$ and $\sigma>0$. Then the control system (7.1), (6.1) exhibits robust stability in the above sense if its parameters satisfy the relationship

$$
\sigma \geq-\frac{v \rho}{2}+\frac{v+\sigma}{2} \sum_{k=1}^{\infty} \frac{\nu_{k} \varphi_{k}\left(x_{0}\right)}{\lambda_{k}}\left(\frac{v}{v+\sigma}\right)^{\frac{\lambda_{k}}{\lambda_{1}}},
$$

where the right-hand side is always positive. If furthermore we have

$$
\sum_{k=2}^{\infty} \frac{\nu_{k} \varphi_{k}\left(x_{0}\right)}{\lambda_{k}} e^{-\lambda_{k} t}<0 \text { for all } t>0
$$

(which is the case of standard parabolic equations in the presence of symmetry, e.g., for heatdiffusion equations on rectangulars, balls, etc.), then the stability condition can be simplified as

$$
\sigma \geq \frac{v}{2 \lambda_{1}}\left(\nu_{1} \varphi_{1}\left(x_{0}\right)-\lambda_{1} \rho\right)
$$

where the right-hand side in (7.4) is always greater than the one in (7.2) whenever $v, \sigma>0$.

Proof. Developing a variational approach to robust stability, consider the following openloop control system on the infinite horizon:

$$
\left\{\begin{array}{l}
\frac{\partial y}{\partial t}+A y=0, \quad x \in \Omega, \quad t \geq 0 \\
y(0, x)=0, \quad x \in \Omega, \\
\left.\left(\alpha y+\frac{\partial y}{\partial \nu_{A}}\right)\right|_{\Sigma}=u(t), \quad t \geq 0
\end{array}\right.
$$

with piecewise constant mixed boundary controls given by

$$
u(t)= \begin{cases}h+\Delta h & \text { if } 0 \leq t \leq \tau \\ h & \text { if } t>\tau\end{cases}
$$


where $h$ and $\Delta h$ are some positive numbers (to be specified later) while $\tau$ is the control switching time to be determined. By Proposition 2.1 we have the representation of the solution $y_{\tau}\left(t, x_{0}\right)$ to mixed boundary problem $(7.5),(7.6)$ :

$$
\begin{aligned}
y_{\tau}\left(t, x_{0}\right) & =\sum_{k=1}^{\infty} \nu_{k} \varphi_{k}\left(x_{0}\right) e^{-\lambda_{k} t}\left(\int_{0}^{\tau}(h+\Delta h) e^{\lambda_{k} \theta} d \theta+\int_{\tau}^{t} h e^{\lambda_{k} \theta} d \theta\right) \\
& =\rho h+\sum_{k=1}^{\infty} \frac{\nu_{k} \varphi_{k}\left(x_{0}\right)}{\lambda_{k}}\left[\Delta h e^{\lambda_{k} \tau}-(h+\Delta h)\right] e^{-\lambda_{k} t} .
\end{aligned}
$$

It is easy to see from (7.7) that

$$
y_{\tau}\left(t, x_{0}\right) \rightarrow \rho h \text { as } t \rightarrow \infty \text { whenever } \tau>0
$$

while the transient $y\left(t, x_{0}\right)$ may intersect the stabilization level (7.8) if the switching time $\tau$ is not properly chosen. We intend to find efficient conditions under which the latter situation does not occur. These conditions, being of their own sake, ensure the required robust stability of the closed-loop system (7.1), (6.1) when the control levels $h$ and $\Delta h$ in (7.6) are specified appropriately.

To proceed, consider the auxiliary dynamic optimization problem for (7.5) on the infinite horizon:

$$
\left\{\begin{array}{l}
\operatorname{minimize} J(\tau):=\rho h-y_{\tau}\left(\tau, x_{0}\right) \\
\text { subject to }(7.5),(7.6), \text { and the state constraint } \\
y_{\tau}\left(t, x_{0}\right)<\rho h \text { for all } t>0 .
\end{array}\right.
$$

The meaning of this problem is to find an optimal switching time $\tau=\bar{\tau}>0$ in (7.6) such that the corresponding trajectory to (7.5) lies strictly below the stabilization level (7.8) for all $t>0$ and that the distance between the stabilization level (7.8) and the switching level $y_{\bar{\tau}}\left(\bar{\tau}, x_{0}\right)$ is minimal. According to the above discussion, solving this problem leads us to required stability conditions.

It follows from the monotonicity property of Theorem 2.2 with respect to controls that the optimal switching time $\bar{\tau}$ is the largest one under which the corresponding transient $y_{\tau}\left(t, x_{0}\right)$ does not intersect the stabilization level $\rho h$ for all $t>0$.

The exact solution to the open-loop control problem (7.9) on the infinite horizon is given in Theorem 5.1(iii). It is provided by the first term rule, i.e., by vanishing the first term in (7.7). Thus we have the rigorously justified formula for the optimal switching time:

$$
\bar{\tau}=\frac{1}{\lambda_{1}} \ln \left(\frac{h+\Delta h}{\Delta h}\right)>0 \text { whenever } v, \sigma>0
$$

and hence the exact optimal value of the cost functional in this problem is computed by:

$$
\bar{\vartheta}:=-\Delta h \rho+(h+\Delta h) \sum_{k=1}^{\infty} \frac{\nu_{k} \varphi_{k}\left(x_{0}\right)}{\lambda_{k}}\left(\frac{\Delta h}{h+\Delta h}\right)^{\frac{\lambda_{k}}{\lambda_{1}}}>0 .
$$

Imposing finally assumption (7.3), we get the feasible first-order approximation

$$
\bar{\vartheta}_{1}:=\Delta h\left[\frac{\nu_{1} \varphi_{1}\left(x_{0}\right)}{\lambda_{1}}-\rho\right]>\bar{\vartheta}>0
$$

to (7.10), which happens to be independent of the control level $h$ in (7.6). 
According to the above description of the instability (in the large) phenomenon, robust stability of the closed-loop system $(7.1),(6.1)$ is ensured if the width of the dead region $2 \sigma$ is not smaller than the value $\bar{\vartheta}$ in (7.10) with $h=\sigma$ and $\Delta h=v$. Substituting these data into (7.10), we arrive at the stability condition (7.2). The first-order approximation condition (7.4) corresponds to substituting the values of $h$ and $\Delta h$ into (7.11) via the sufficient stability requirement $2 \sigma \geq \bar{\vartheta}_{1}$.

\section{References}

[1] Agmon, S., Lectures on elliptic boundary value problems, Van Nostrand (Princeton, New Jersey, 1965).

[2] Başar, T., Bernhard, P., $H_{\infty}$-optimal control and related minimax design problems, Birkhäuser (Boston, Massachusetts, 1995).

[3] Keulen, B. van, $H_{\infty}$-control for distributed parameter systems: A state-space approach, Birkhäuser, (Boston, Massachusetts, 1993).

[4] Krasovskii, N. N., Subbotin, A. I., Game-theoretical control problems, Springer (New York, 1988).

[5] Ladyzhenskaya, O. A., Solonnikov, A. I., Uraltseva, N. N., Linear and quasilinear equations of parabolic type, American Mathematical Society (Providence, Rhode Island, 1968).

[6] Lions, J.-L., Optimal control of systems governed by partial differential equations, Springer (Berlin, 1971).

[7] Mordukhovich, B. S., Optimal control of the groundwater regime on engineering reclamation systems, Water Resources 12, 244 (1986).

[8] Mordukhovich, B. S., Minimax design for a class of distributed parameter systems, Autom. Remote Control 50, 262 (1990).

[9] Mordukhovich, B. S., Variational analysis and generalized differentiation, II: Applications, Springer (Berlin, 2006).

[10] Mordukhovich, B. S., Suboptimal feedback control design of constrained parabolic sytems in uncertainty conditions, SIAM J. Control Optim., to appear.

[11] Mordukhovich, B. S., Seidman, T. I., Asymmetric games for convolution systems with applications to feedback control of constrained parabolic equations, J. Math. Anal. Appl., to appear.

[12] Mordukhovich, B. S., Zhang, K., Robust suboptimal control of constrained parabolic systems under uncertaintly conditions, in Dynamic and Control (Leitmann, G., Udwadia, F. E., Kryazhimskii, A. V., eds.), Gordon and Breach (Amsterdam, 1999).

[13] Pontryagin, L. S., Boltyanskii, V. G., Gamkrelidze, R. V., Mishchenko, E. F., The mathematical theory of optimal processes, Wiley (New York, 1962). 